Revta brasil. Bot., São Paulo, V.23, n.4, p.443-449, dez. 2000

\title{
Respostas de indivíduos jovens de Tibouchina pulchra Cogn. à poluição aérea de Cubatão, SP: fotossíntese líquida, crescimento e química foliar
}

\author{
REGINA MARIA DE MORAES ${ }^{1,4}$, WELINGTON BRAZ CARVALHO DELITTI ${ }^{2} \mathrm{e}$ \\ JOSÉ ANTÔNIO PROENÇA VIEIRA DE MORAES ${ }^{3}$
}

(recebido em 22 de dezembro de 1999; aceito em 23 de agosto de 2000)

\begin{abstract}
Responses of saplings of Tibouchina pulchra Cogn. to air pollution in Cubatão, SP: net photosynthesis, growth and leaf chemistry). Saplings of Tibouchina pulchra, cultivated on standardised conditions, were placed in three sites at the Cubatão region to study the effects of the air pollutants on photosynthesis, growth and leaf concentrations of ascorbic acid, chlorophyll, nitrogen, sulfur and fluoride. The study areas were: Pilões river valley (RP), considered as the reference plot; Caminho do Mar (CM), near the petrochemical industries; and Mogi river valley (VM), near metallurgical and fertilizer industries. Four plants remained in each plot during six months. Compared to the reference plot $\left(\mathrm{RP}-9.81 \mu \mathrm{mol} . \mathrm{m}^{-2} . \mathrm{s}^{-1}\right)$, net photosynthesis was reduced $(\mathrm{p}<0.05)$ in plants at the VM site $\left(8.02 \mu \mathrm{mol} . \mathrm{m}^{-2} . \mathrm{s}^{-1}\right)$, and it was not altered in the other area $\left(\mathrm{CM}-9.29 \mu \mathrm{mol} . \mathrm{m}^{-2} . \mathrm{s}^{-1}\right)$. Plants grown in VM showed reduction in height and the amount of ascorbic acid, alterations on the pattern of biomass distribution in the plant, increased concentrations of chlorophyll a and b, fluoride, nitrogen and sulfur. Plants exposed in CM showed reduction in both height and diameter, and enhanced concentrations of chlorophyll a and $\mathrm{b}$, fluoride, $\mathrm{N}$ and $\mathrm{S}$. The results indicate that pollutant concentrations have still been at phytotoxic levels in VM and CM and this problem was more intensive in VM, during the exposure period.
\end{abstract}

RESUMO - (Respostas de indivíduos jovens de Tibouchina pulchra Cogn. à poluição aérea de Cubatão, SP: fotossíntese, crescimento e química foliar). Indivíduos jovens de Tibouchina pulchra, cultivados em condições padronizadas, foram colocados em três regiões de Cubatão para estudo dos possíveis efeitos dos poluentes aéreos sobre a fotossíntese, o crescimento e as concentrações foliares de ácido ascórbico, clorofila, nitrogênio, enxofre e flúor. As áreas de estudo foram: vale do rio Pilões (RP), considerada área de referência; Caminho do Mar (CM), próxima a indústrias petroquímicas e vale do rio Mogi (VM), próxima a indústrias siderúrgicas e de fertilizantes. Quatro plantas permaneceram em cada área por um período de seis meses. Em comparação com as plantas mantidas na área de referência (RP - 9,81 $\left.\mu \mathrm{mol} \cdot \mathrm{m}^{-2} \cdot \mathrm{s}^{-1}\right)$, a fotossíntese líquida foi reduzida $(\mathrm{p}<0,05)$ nas plantas que foram mantidas em VM $\left(8,02 \mu \mathrm{mol} \cdot \mathrm{m}^{-2} \cdot \mathrm{s}^{-1}\right), \mathrm{e}$ não foi alterada em $\mathrm{CM}\left(9,29 \mu \mathrm{mol} \cdot \mathrm{m}^{-2} \cdot \mathrm{s}^{-1}\right)$. Em VM, as plantas apresentaram redução do crescimento em altura e do conteúdo de ácido ascórbico, alteração no padrão de distribuição de biomassa entre as partes da planta e aumento das concentrações foliares de clorofila a e b, flúor, nitrogênio e enxofre. As plantas de CM apresentaram redução no crescimento em altura e diâmetro, aumento nas concentrações foliares de clorofila a e b, flúor, nitrogênio e enxofre. Os resultados obtidos indicam que as concentrações de poluentes ainda se encontram em níveis fitotóxicos em CM e VM, tendo sido mais altas em VM, durante o período de exposição.

Key words - Air pollution, Tibouchina pulchra, Cubatão, photosynthesis

\section{Introdução}

O monitoramento biológico tem recebido recentemente considerável atenção por parte de ecólogos e toxicologistas, constituindo-se em poderoso instrumento de avaliação da saúde ambiental, particularmente em ambientes impactados pela poluição. Ele se refere ao uso de organismos vivos na determinação das condições ambientais, envolvendo o con-

1. Instituto de Botânica, Caixa Postal 4005, 01061-970 São Paulo, SP, Brasil.

2. Departamento de Ecologia Geral, Universidade de São Paulo, Caixa Postal 11461, 05422-970 São Paulo, SP, Brasil.

3. Departamento de Botânica, Universidade Federal de São Carlos, Caixa Postal 676, 13565-905 São Carlos, SP, Brasil.

4. Autor para correspondência: rmoraes@smtpgw.ibot.sp.gov.br ceito de que respostas biológicas selecionadas podem proporcionar indicações seguras de exposição do organismo ao estresse (Shugart 1994).

A fotossíntese é bastante sensível a condições ambientais adversas. Numerosos estudos sobre o declínio de florestas têm comprovado a ação deletéria dos poluentes aéreos sobre aquele processo (Pfanz et al. 1994, Reich et al. 1994). Em nível de organismo, a fotossíntese é um dos primeiros processos alterado por ação de poluentes, ocorrendo sua redução, via de regra, antes que a planta apresente sintomas visíveis (Mooney \& Winner 1988, Treshow \& Anderson 1991).

A floresta Atlântica que recobre as encostas da Serra do Mar, na região de Cubatão, SP, tem sido afetada por poluentes aéreos emitidos pelo complexo industrial lá instalado há algumas décadas e, apesar da redução das emissões (Alonso \& Godinho 1992), 
o impacto sobre a vegetação ainda persiste (Domingos et al. 1998, Klumpp et al. 1998). O objetivo deste estudo foi verificar os efeitos de poluentes aéreos sobre a fotossíntese líquida, crescimento e alguns parâmetros químicos em indivíduos jovens de Tibouchina pulchra Cogn., o manacá-da-serra, utilizada como indicador biológico. T. pulchra pertence à família Melastomataceae, é arbórea, nativa, pioneira e está distribuída por toda a região de estudo, sendo importante na definição da fisionomia e estrutura da vegetação (Leitão-Filho et al. 1993). Segundo Klumpp et al. (1996), por ser uma espécie dominante dessa formação, T. pulchra pode ser considerada uma boa representante da floresta Atlântica para biomonitoramento ambiental, permitindo a transferência dos resultados obtidos para a vegetação natural.

\section{Material e métodos}

O município de Cubatão situa-se a $23^{\circ} 45^{\prime}-23^{\circ} 55^{\prime} \mathrm{S}$ e $46^{\circ} 21^{\prime}-46^{\circ} 30^{\prime} \mathrm{W}$, em uma planície litorânea estreita envolvida pelas escarpas da Serra do Mar a norte, oeste e leste.

Apresenta clima tropical super-úmido sem estiagem, com nebulosidade, umidade relativa e precipitação altas $(2600 \mathrm{~mm}$ anuais). A temperatura média anual é de $23^{\circ} \mathrm{C}$. A floresta Atlântica nessa região é secundária e apresenta redução de sua diversidade e simplificação de sua estrutura (Leitão-Filho et al. 1993).

Cubatão abriga uma grande concentração de indústrias químicas, petroquímicas, siderúrgicas e de fertilizantes, totalizando 110 unidades de produção e cerca de 260 fontes de emissão de poluentes aéreos, entre os quais, fluoretos gasosos, amônia, dióxido de enxofre, óxidos de nitrogênio, compostos orgânicos e materiais particulados. A qualidade do ar e o impacto dos poluentes sobre a vegetação não são homogêneos, variando de acordo com os padrões de circulação e estagnação das massas de ar e as características orográficas da região (Jaeschke 1997). Diante disso, foram escolhidas três áreas experimentais que apresentam, segundo Jaeschke (1997) e Klumpp et al. (1997), diferentes tipos e níveis de poluição aérea:

Rio Pilões - RP - (40 m.s.m.) - área de referência, localizada em área de floresta, no vale do rio Pilões, ao abrigo dos ventos. Sua vegetação aparentemente não apresenta danos (Leitão-Filho et al. 1993).

Caminho do Mar - CM - (80 m.s.m.) - situada em uma antiga rodovia que fazia a ligação entre a cidade de São Paulo e o litoral. Em sua base, estão instaladas indústrias petroquímicas cujas emissões de poluentes têm afetado severamente a vegetação do entorno (Klumpp et al. 1997). Os principais poluentes encontrados nessa região são compostos orgânicos e poluentes secundários, como ozônio e nitrato de peroxiacetila (PAN), além de óxidos de enxofre e de nitrogênio.

Vale do rio Mogi - VM - (20 m.s.m.) - situada na entrada do vale do rio Mogi por onde são canalizados os ventos que carregam poluentes oriundos de indústrias de fertilizantes, cimento e side- rurgia, causando, também, danos severos à vegetação (LeitãoFilho et al. 1993). Fluoretos gasosos e materiais particulados constituem os principais poluentes dessa região, seguidos por dióxido de enxofre, amônia e óxidos de nitrogênio.

Plantas jovens de T. pulchra, com cerca de $30 \mathrm{~cm}$ de altura e mesma idade, foram adquiridas no viveiro da Companhia Energética de São Paulo (CESP), em Paraibuna, SP, e plantadas em vasos plásticos $(10 \mathrm{~L})$, utilizando solo de floresta coletado na mata da sede do Instituto de Botânica, em São Paulo. A exposição foi feita colocando-se os vasos com as plantas dentro de telados construídos com armação de ferro e cobertos com sombrite 50\%. Os vasos ficaram sobre caixas utilizadas como reservatórios de água $(50 \mathrm{~L})$, cobertas com grades de metal. Os vasos possuíam seis cordas de náilon, que ficavam mergulhadas na água, garantindo suprimento hídrico contínuo. As plantas ficaram expostas de março a setembro de 1997, período que compreende os meses de pior qualidade do ar em Cubatão (CETESB 1999).

Para a realização das medidas de trocas gasosas, as plantas das áreas poluídas foram levadas para a área de referência para que as condições ambientais durante as medições fossem padronizadas. As trocas gasosas foram determinadas em condições de campo, entre 9:00 e 10:30 h, com um sistema portátil de análise de gases por infravermelho (LCA-4, ADC, Hoddesdon, Reino Unido), ligado a uma câmara foliar de $11 \mathrm{~cm}^{2}$ (PCL-N) com controle de temperatura $\left(25^{\circ} \mathrm{C}\right)$ e irradiância (densidade de fluxo de fótons fotossinteticamente ativos de $\left.900 \mu \mathrm{mol} . \mathrm{m}^{-2} . \mathrm{s}^{-1}\right)$ e monitoramento da umidade relativa do ar (em torno de 55\%) e da concentração de $\mathrm{CO}_{2}$ (entre 340 e $360 \mu \mathrm{mol} . \mathrm{m}^{-2}$. $\mathrm{s}^{-1}$ ). As taxas de fotossíntese líquida, de transpiração e de condutância estomática foram determinadas em folhas completamente expandidas do terceiro nó, sendo efetuados quatro registros por folha, em quatro plantas por área.

No laboratório, após as medidas de campo, foram determinados: presença de sintomas visíveis (cloroses e necroses); incremento em diâmetro basal e altura da gema apical; biomassa de folhas, caules e raízes; razão raiz/parte aérea; razão folhas/planta inteira; razão raízes/planta inteira; concentração foliar de ácido ascórbico (Keller \& Schweizer 1977), de clorofila a e b (Barnes et al. 1992), de nutrientes (Zagatto et al. 1981) e de flúor (AOAC 1975).

Foi realizada análise de variância (ANOVA) para verificar a existência de diferenças entre as médias dos parâmetros analisados em relação as áreas estudadas. Nos casos em que ocorreram diferenças, as médias foram discriminadas por meio de teste de comparações múltiplas (Tukey).

\section{Resultados e Discussão}

A taxa de fotossíntese líquida foi menor nas plantas expostas ao ambiente do vale do rio Mogi (VM), 8,02 $\mu \mathrm{mol} \cdot \mathrm{m}^{-2} \cdot \mathrm{s}^{-1},(\mathrm{p}<0,05)$, e não diferiu entre as plantas que permaneceram no Caminho do Mar $(\mathrm{CM})$ e vale do rio Pilões (RP), 9,27 e $9,92 \mu \mathrm{mol} . \mathrm{m}^{-2} \cdot \mathrm{s}^{-1}$, respectivamente (tabela 1$)$. A fotossíntese é um processo sensível à poluição aérea e sua redução tem sido constatada com frequiência em plantas ocorrentes ou introduzidas em locais poluí- 
Tabela 1. Valores médios e erro padrão $(\mathrm{n}=4)$ das taxas de fotossíntese líquida, condutância estomática e transpiração em indivíduos jovens de T. pulchra após seis meses de exposição no vale do rio Pilões (RP), no Caminho do Mar (CM) e no vale do rio Mogi (VM), em Cubatão, SP. Valores seguidos por letras diferentes em cada linha são significativamente diferentes $(\mathrm{p}<0,05)$.

\begin{tabular}{lccc}
\hline & $\mathrm{RP}$ & $\mathrm{CM}$ & $\mathrm{VM}$ \\
\hline Fotossíntese líquida $\left(\mu \mathrm{mol} \cdot \mathrm{m}^{-2} \cdot \mathrm{s}^{-1}\right)$ & $9,81 \pm 0,27 \mathrm{a}$ & $9,29 \pm 0,20 \mathrm{a}$ & $8,02 \pm 0,56 \mathrm{~b}$ \\
Condutância estomática $\left(\mu \mathrm{mol} \cdot \mathrm{m}^{-2} \cdot \mathrm{s}^{-1}\right)$ & $0,30 \pm 0,05 \mathrm{a}$ & $0,35 \pm 0,04 \mathrm{a}$ & $0,24 \pm 0,03 \mathrm{a}$ \\
Transpiração $\left(\mu \mathrm{mol} \cdot \mathrm{m}^{-2} \cdot \mathrm{s}^{-1}\right)$ & $1,60 \pm 0,18 \mathrm{a}$ & $1,67 \pm 0,12 \mathrm{a}$ & $1,55 \pm 0,18 \mathrm{a}$ \\
\hline
\end{tabular}

dos (Inoue \& Reissmann 1994, Pandey \& Agrawal 1994, Pfanz et al. 1994, Reich et al. 1994). Segundo Pell et al. (1994), o processo fotossintético pode ser afetado pela ocorrência de danos envolvendo os movimentos estomáticos, a coleta de luz ou a etapa bioquímica de fixação do $\mathrm{CO}_{2}$. Como neste estudo não foram observadas diferenças nas taxas de condutância estomática e de transpiração (tabela 1), acredita-se que a redução da fotossíntese tenha sido causada por outros fatores, envolvendo alterações na coleta de luz ou na quantidade e atividade da ribulose-1,5-bisfosfato carboxilase-oxigenase (RUBISCO). Resultados semelhantes foram observados por Clark et al. (1996), Renaud et al. (1997) e Shan (1998). Contudo, como a atmosfera de Cubatão é contaminada por combinações de vários poluentes, cujas concentrações variam no tempo e no espaço, é difícil a avaliação precisa do sítio de ação dos poluentes sobre a fotossíntese em $T$. pulchra.
Não foi constatada a ocorrência de sintomas visíveis, como necroses ou cloroses, nas folhas das plantas que foram expostas nas três áreas de estudo ao final do experimento, mesmo naquelas que ficaram expostas ao ambiente mais poluído (VM) e que apresentaram redução na fotossíntese líquida. Esse tipo de resposta é freqüente quando as concentrações de poluentes são relativamente baixas, causando alteração em processos fisiológicos sem que ocorra a manifestação de sintomas visíveis (Samuelson \& Edwards 1993, Shan 1998).

Quando comparadas às plantas da área de referência (RP), aquelas mantidas em CM apresentaram menor incremento em altura e diâmetro, e as do vale do rio Mogi, apenas menor incremento em altura (tabela 2). Domingos (1998) também observou redução do crescimento em altura em plantas jovens de T. pulchra expostas às condições ambientais do Caminho do Mar. Pandey \& Agrawal (1994) observaram o mesmo em plantas utilizadas na arborização

Tabela 2. Valores médios e erro padrão $(n=4)$ de incremento em altura e diâmetro, biomassa de folhas, caules, raízes e total, razões entre as biomassas de folhas/planta inteira, de raízes/planta inteira e de raízes/parte aérea e massa foliar específica, em indivíduos jovens de T. pulchra após seis meses de exposição no vale do rio Pilões (RP), no Caminho do Mar (CM) e no vale do rio Mogi (VM), em Cubatão, SP. Valores seguidos por letras diferentes em cada linha são significativamente diferentes $(\mathrm{p}<0,05)$.

\begin{tabular}{lccc}
\hline & RP & CM & VM \\
\hline Incremento em altura (cm) & $35,0 \pm 1,75 \mathrm{a}$ & $28,5 \pm 1,47 \mathrm{~b}$ & $26,4 \pm 1,54 \mathrm{~b}$ \\
Incremento em diâmetro (cm) & $0,58 \pm 0,04 \mathrm{a}$ & $0,51 \pm 0,04 \mathrm{~b}$ & $0,60 \pm 0,01 \mathrm{a}$ \\
Biomassa de folhas (g de massa seca) & $16,15 \pm 1,87 \mathrm{a}$ & $14,51 \pm 1,69 \mathrm{a}$ & $19,94 \pm 2,28 \mathrm{a}$ \\
Biomassa de caules (g de massa seca) & $13,99 \pm 1,17 \mathrm{a}$ & $12,19 \pm 0,64 \mathrm{a}$ & $15,30 \pm 1,50 \mathrm{a}$ \\
Biomassa de raízes (g de massa seca) & $20,16 \pm 3,71 \mathrm{a}$ & $15,02 \pm 2,20 \mathrm{a}$ & $15,97 \pm 2,28 \mathrm{a}$ \\
Biomassa da planta inteira (g de massa seca) & $50,29 \pm 5,84 \mathrm{a}$ & $41,72 \pm 4,18 \mathrm{a}$ & $51,21 \pm 5,61 \mathrm{a}$ \\
Folhas/Planta inteira & $0,32 \pm 0,03 \mathrm{~b}$ & $0,33 \pm 0,01 \mathrm{ab}$ & $0,39 \pm 0,01 \mathrm{a}$ \\
Raízes/Planta inteira & $0,40 \pm 0,03 \mathrm{a}$ & $0,36 \pm 0,02 \mathrm{ab}$ & $0,31 \pm 0,02 \mathrm{~b}$ \\
Raízes/Parte Aérea & $0,67 \pm 0,08 \mathrm{a}$ & $0,56 \pm 0,04 \mathrm{ab}$ & $0,45 \pm 0,04 \mathrm{~b}$ \\
Massa foliar específica (mg.cm ${ }^{-2}$ ) & $5,83 \pm 0,80 \mathrm{a}$ & $6,02 \pm 0,26 \mathrm{a}$ & $5,50 \pm 0,25 \mathrm{a}$ \\
\hline
\end{tabular}


de áreas poluídas, na Índia. No Caminho do Mar, é freqüente a ocorrência de episódios fitotóxicos de poluentes como $\mathrm{O}_{3}$ e PAN (Klumpp et al. 1994, Jaeschke 1997), os quais têm sido relacionados com a redução de crescimento (Treshow \& Anderson 1991, Schmieden \& Wild 1995), o que poderia também estar relacionado aos resultados obtidos no presente estudo.

Não foram verificadas diferenças significativas quanto à massa foliar específica e os parâmetros relativos à produção de biomassa (tabela 2), mesmo nas plantas que permaneceram em VM, nas quais a fotossíntese foi reduzida. É possível que o aporte adicional de nutrientes, como nitrogênio e fósforo, provenientes das emissões das indústrias de fertilizantes existentes na região, tenha compensado o efeito inibitório da poluição, como mencionado por Klumpp et al. (1998). Entretanto, as razões entre as biomassas de folhas/planta inteira, raízes/planta inteira e raízes/parte aérea evidenciaram alterações no padrão de partição de carbono entre os diferentes órgãos dessas plantas. Segundo Mooney \& Winner (1988), embora a redução da fotossíntese possa resultar em redução de crescimento, a relação entre esses dois parâmetros não é necessariamente linear, pois a exposição a poluentes causa freqüentemente alterações na distribuição do carbono entre os órgãos da planta, ocorrendo maior produção de biomassa foliar, em detrimento das raízes (Klumpp et al. 1997, Domingos 1998, Klumpp et al. 1998). Esse fato é evidenciado pela correlação positiva entre a fotossíntese e as razões raiz/parte aérea e raiz/planta inteira $(r=0,69$ e $r=0,73$, respectivamente, $p=0,01)$, e negativa entre a fotossíntese e a razão folhas/planta inteira $(r=-0,77, p=0,003)$. De acordo com Amthor $\&$ McCree (1990), em plantas expostas a poluentes aéreos que apresentam redução da fotossíntese, a produção de biomassa foliar é a menos afetada por- que a poluição inibe a translocação de carboidratos das folhas para as raízes (Rennenberg et al. 1996) alterando, portanto, as razões entre a biomassa de partes da planta em relação à planta inteira.

As concentrações foliares de clorofila $a$ e $b$ foram mais altas nas plantas que permaneceram nas áreas sob poluição (tabela 3). Este fato é devido possivelmente à presença de fontes emissoras de $\mathrm{NO}_{\mathrm{x}} \mathrm{e} \mathrm{NH}_{4}$ nessas áreas pois, como se pode constatar na tabela 4, a concentração foliar de nitrogênio foi consideravelmente maior nas áreas poluídas. $\mathrm{O} \mathrm{N}$ proveniente das fontes poluidoras pode ter sido utilizado na síntese de clorofila, hipótese reforçada pelo valor alto de correlação de Pearson entre a concentração de nitrogênio e o conteúdo total de clorofila ( $\mathrm{r}$ $=0,91, p<0,0001)$ e pela análise de regressão linear, onde, clorofila $=-0,96+(0,20 \mathrm{~N})$, com $\mathrm{r}=0,87 \mathrm{e}$ $\mathrm{p}<0,0001$. O incremento do conteúdo de clorofila, entretanto, não impediu a redução da fotossíntese nas plantas que permaneceram no vale do Mogi, não havendo correlação entre essas duas variáveis nessas plantas. Shan (1998) verificou que folhas de Pinus densiflora Sieb. expostas a um tratamento simulando chuva ácida, apresentaram aumento do teor de clorofila, mas a taxa de fotossíntese líquida foi reduzida, indicando diminuição da eficiência de uso do pigmento na fotossíntese. Deste modo, pode-se concluir que, em plantas expostas à presença de óxidos de nitrogênio, pode ocorrer incremento da concentração de clorofila sem que isso implique necessariamente em aumento na assimilação do carbono.

A concentração foliar de ácido ascórbico foi reduzida nas plantas que permaneceram em VM (tabela 3). O ácido ascórbico é um antioxidante natural capaz de manter a estabilidade das membranas celulares em plantas sob estresse causado por poluentes e de neutralizar radicais livres citotóxicos (Pandey \& Agrawal 1994). A redução de sua con-

Tabela 3. Valores médios e erro padrão $(n=4)$ das concentrações foliares de clorofila a, clorofila b, flúor e ácido ascórbico em indivíduos jovens de T. pulchra após seis meses de exposição no vale do rio Pilões (RP), no Caminho do Mar (CM) e no vale do rio Mogi (VM), em Cubatão, SP. Valores seguidos por letras diferentes em cada linha são significativamente diferentes $(\mathrm{p}<0,05)$.

\begin{tabular}{lccc}
\hline & $\mathrm{RP}$ & $\mathrm{CM}$ & $\mathrm{VM}$ \\
\hline Clorofila a $\left(\mu \mathrm{g} \cdot \mathrm{g}^{-1}\right.$ de massa seca) & $2,15 \pm 0,64 \mathrm{~b}$ & $2,84 \pm 0,16 \mathrm{a}$ & $2,82 \pm 0,19 \mathrm{a}$ \\
Clorofila b $\left(\mu \mathrm{g} \cdot \mathrm{g}^{-1}\right.$ de massa seca) & $0,57 \pm 0,19 \mathrm{~b}$ & $0,89 \pm 0,19 \mathrm{a}$ & $0,88 \pm 0,18 \mathrm{a}$ \\
Ácido ascórbico (mg.g $\mathrm{g}^{-1}$ de massa seca) & $1,68 \pm 0,22 \mathrm{a}$ & $1,23 \pm 0,21 \mathrm{ab}$ & $0,79 \pm 0,27 \mathrm{~b}$ \\
Flúor $\left(\mu \mathrm{g} \cdot \mathrm{g}^{-1}\right.$ de massa seca) & $23,37 \pm 1,83 \mathrm{c}$ & $35,07 \pm 3,24 \mathrm{~b}$ & $209,17 \pm 5,38 \mathrm{a}$ \\
\hline
\end{tabular}


Tabela 4. Valores médios e erro padrão $(\mathrm{n}=4)$ das concentrações foliares de nitrogênio, enxofre, fósforo, cálcio, magnésio e potássio (mg. $\mathrm{g}^{-1}$ de massa seca) em indivíduos jovens de T. pulchra após seis meses de exposição no vale do rio Pilões (RP), no Caminho do Mar (CM) e no vale do rio Mogi (VM), em Cubatão, SP. Valores seguidos por letras diferentes em cada linha são significativamente diferentes $(\mathrm{p}<0,05)$.

\begin{tabular}{lccc}
\hline Elementos & RP & CM & VM \\
\hline Nitrogênio & $18,45 \pm 1,49 \mathrm{~b}$ & $23,78 \pm 0,27 \mathrm{a}$ & $24,71 \pm 0,66 \mathrm{a}$ \\
Enxofre & $6,25 \pm 0,18 \mathrm{~b}$ & $7,68 \pm 0,24 \mathrm{a}$ & $8,23 \pm 0,25 \mathrm{a}$ \\
Fósforo & $0,98 \pm 0,08 \mathrm{~b}$ & $1,18 \pm 0,06 \mathrm{ab}$ & $1,40 \pm 0,08 \mathrm{a}$ \\
Cálcio & $19,58 \pm 1,13 \mathrm{a}$ & $16,68 \pm 0,34 \mathrm{a}$ & $18,50 \pm 1,03 \mathrm{a}$ \\
Magnésio & $3,63 \pm 0,23 \mathrm{a}$ & $3,78 \pm 0,14 \mathrm{a}$ & $3,85 \pm 0,35 \mathrm{a}$ \\
Potássio & $6,10 \pm 0,28 \mathrm{a}$ & $7,33 \pm 0,36 \mathrm{a}$ & $6,58 \pm 0,51 \mathrm{a}$ \\
\hline
\end{tabular}

centração em plantas sob efeito de poluição pode indicar redução de sua síntese ou aumento do consumo em processos de desintoxicação. Obteve-se correlação significativa entre a concentração de ácido ascórbico e a fotossíntese líquida ( $\mathrm{r}=0,64$, $\mathrm{p}=0,02)$. A redução da concentração de ácido ascórbico nas plantas de VM poderia estar relacionada ao aumento nas concentrações foliares de flúor, nitrogênio e enxofre, uma vez que foram verificadas correlações significativas entre esses compostos $(\mathrm{r}=-0,76, \mathrm{p}=0,003 ; \mathrm{r}=-0,75, \mathrm{p}=0,005 ; \mathrm{r}=-0,83$, $\mathrm{p}<0,000$, para $\mathrm{F}^{-}, \mathrm{N}$ e $\mathrm{S}$, respectivamente). Klumpp et al. (1998) e Domingos (1998) também verificaram redução do conteúdo de ácido ascórbico em espécies arbóreas tropicais cultivadas na região do vale do rio Mogi. Segundo Guzy \& Heath (1993), quanto maior a concentração foliar de ácido ascórbico, menor é a sensibilidade da planta à poluição. Assim, a redução de seus teores nas plantas expostas em VM corrobora a hipótese de que as mesmas estiveram sob estresse.

As plantas que permaneceram em VM apresentaram grande acúmulo de flúor em seus tecidos foliares, com uma concentração foliar nove vezes superior à das plantas controle (tabela 3). As folhas das plantas que ficaram expostas em CM também apresentaram acúmulo de flúor, porém menor do que o observado em VM, ou seja, 1,5 vezes o valor obtido na área controle. Resultados semelhantes foram obtidos em outros estudos conduzidos em Cubatão com indivíduos jovens de T. pulchra (Klumpp et al. 1996, Domingos et al. 1998, Klumpp et al. 1998). O acúmulo de flúor nos tecidos foliares das plantas expostas no vale do Mogi pode ser atribuído aos níveis ainda altos de fluoretos no ar, emitidos pelas indús- trias de fertilizantes nesse local. A concentração foliar de flúor apresentou correlação negativa com a fotossíntese líquida $(r=-0,77, p=0,003)$, o que era esperado, pois esse elemento é tóxico e, dentre todos os poluentes gasosos, é capaz de inibir a fotossíntese quando em concentrações mínimas (Darral 1989).

As plantas que foram expostas em VM apresentaram acúmulo de $\mathrm{N}, \mathrm{S}$ e $\mathrm{P}$, e aquelas que permaneceram em CM, de $\mathrm{N}$ e $\mathrm{S}$. As concentrações dos demais nutrientes analisados foram semelhantes nas plantas expostas nas três áreas de estudo (tabela 4). Enriquecimento em $\mathrm{N}$ em plantas do Caminho do Mar e do vale do rio Mogi também foi observado por Domingos et al. (1998) e Furlan et al. (1999). Este enriquecimento poderia ser atribuído às emissões de $\mathrm{NO}_{x}$ pela refinaria localizada no Caminho do Mar e pelas indústrias de fertilizantes do vale do rio Mogi. $\mathrm{O}$ aumento das concentrações foliares de $\mathrm{N}$ pode, também, ser induzido pela ação de poluentes não nitrogenados, como $\mathrm{O}_{3}$ e $\mathrm{SO}_{2}$ (Lea et al. 1996), os quais estão presentes na atmosfera de Cubatão. A concentração de $\mathrm{N}$ não apresentou correlação com a fotossíntese líquida. $\mathrm{O}$ acúmulo de $\mathrm{S}$ nas plantas expostas em CM e VM também foi verificado em outros estudos de biomonitoramento (Domingos et al. 1998, Klumpp et al. 1998, Szabo 1999). Segundo Jaeschke (1997), o ar destas regiões apresenta-se contaminado por compostos sulfurosos. $\mathrm{O} \mathrm{SO}_{2}$ é um dos poluentes aéreos mais nocivos às plantas, possuindo características oxidativas e provocando o aumento da acidificação dos fluídos celulares (Rennenberg \& Herschbach 1996). A concentração de S apresentou correlação negativa com a fotossíntese líquida $(r=-0,61, p=0,03)$, evidenciando seu efeito 
Tabela 5. Valores médios e erro padrão $(n=4)$ dos conteúdos foliares de nitrogênio, enxofre, fósforo, cálcio, magnésio, potássio e da somatória de nutrientes (mg), em indivíduos jovens de T. pulchra após seis meses de exposição no vale do rio Pilões (RP), no Caminho do Mar (CM) e no vale do rio Mogi (VM), em Cubatão, SP. Valores seguidos por letras diferentes em cada linha são significativamente diferentes $(\mathrm{p}<0,05)$.

\begin{tabular}{lccc}
\hline Elementos & RP & CM & VM \\
\hline Nitrogênio & $292,24 \pm 1,94 \mathrm{~b}$ & $344,79 \pm 2,77 \mathrm{~b}$ & $491,02 \pm 3,11 \mathrm{a}$ \\
Enxofre & $100,26 \pm 1,37 \mathrm{~b}$ & $111,42 \pm 1,63 \mathrm{~b}$ & $163,67 \pm 1,86 \mathrm{a}$ \\
Fósforo & $15,51 \pm 0,51 \mathrm{~b}$ & $16,85 \pm 0,50 \mathrm{~b}$ & $27,82 \pm 0,78 \mathrm{a}$ \\
Cálcio & $318,37 \pm 3,06 \mathrm{a}$ & $242,63 \pm 2,43 \mathrm{a}$ & $370,61 \pm 3,16 \mathrm{a}$ \\
Magnésio & $58,45 \pm 1,23 \mathrm{a}$ & $54,57 \pm 1,07 \mathrm{a}$ & $76,16 \pm 1,28 \mathrm{a}$ \\
Potássio & $98,05 \pm 1,45 \mathrm{a}$ & $106,44 \pm 1,67 \mathrm{a}$ & $128,71 \pm 1,05 \mathrm{a}$ \\
Total & $882,89 \pm 4,11 \mathrm{~b}$ & $876,79 \pm 4,48 \mathrm{~b}$ & $1257,98 \pm 4,98 \mathrm{a}$ \\
\hline
\end{tabular}

deletério nas plantas. Observou-se, ainda, que as plantas de VM apresentaram acúmulo foliar de $\mathrm{P}$ (tabela 4), o que também pode ter sido causado pela proximidade das indústrias de fertilizantes. Os conteúdos foliares de N, S e P foram maiores nas plantas mantidas em VM, acompanhando os resultados relativos às concentrações desses elementos, uma vez que não houve diferenças quanto a biomassa foliar (tabela 5).

Assim, decorridos seis meses de exposição, verificou-se que as plantas do VM foram as que apresentaram maiores concentrações e conteúdos de $\mathrm{N}, \mathrm{P}$ e S enquanto que os demais macronutrientes não diferiram do controle. Apresentaram, também, as maiores concentrações de clorofila. Mesmo assim, a taxa de fotossíntese líquida foi menor e a produção de biomassa não variou em relação às plantas que permaneceram na área sem poluição, RP, indicando redução na eficiência do uso de nutrientes. Estes resultados, juntamente com os referentes ao acúmulo foliar de flúor e à redução da concentração de ácido ascórbico, evidenciam que estas plantas, apesar de não apresentarem danos visíveis, foram submetidas a condições ambientais estressantes e que, portanto, os níveis de poluição aérea no vale do rio Mogi ainda são fitotóxicos.

Agradecimentos - Os autores agradecem à Fundação de Amparo à Pesquisa do Estado de São Paulo (processo n. 97/12163-7) pelo auxílio concedido.

\section{Referências bibliográficas}

ALONSO, C.D. \& GODINHO, R. 1992. A evolução da qualidade do ar em Cubatão. Química Nova 15:126-136.

AMTHOR, J.S. \& McCREE, K. 1990. Carbon balance of stressed plants: a conceptual model for integrating research results. In Stress responses in plants: adaptation and acclimation mechanisms (R.G. Alscher \& J.R. Cumming, eds.). Wiley-Liss, New York, p.1-15.

AOAC (Association of Official Analytical Chemist). 1975. Fluoride potentiometric method - official first action. Journal of the Association of Official Analytical Chemist 58:384-385

BARNES, J., BALANGUER, L., MANRIQUE, E., ELVIRA, S. \& DAVISON, A.W. 1992. A reappraisal of the use of DMSO for the extraction and determination of chlorophylls $a$ and $b$ in lichens and higher plants. Environmental and Experimental Botany 32:85-100.

CETESB - COMPANHIA DE TECNOLOGIA DE SANEAMENTO AMBIENTAL. 1999. Relatório anual de qualidade do ar - 1998. Série Relatórios, CETESB, São Paulo.

CLARK, C.S., WEBER, J.A., LEE, E.H. \& HOGSETT, W.E. 1996. Reductions in gas exchange of Populus tremuloides caused by leaf aging and ozone exposure. Canadian Journal of Forest Research 26:1384-1391.

DARRALL, N.M. 1989. The effect of air pollutants on physiological processes in plants. Plant, Cell and Environment 12:1-30.

DOMINGOS, M. 1998. Biomonitoramento da fitotoxidade da poluição aérea e da contaminação do solo na região do complexo industrial de Cubatão, São Paulo, utilizando Tibouchina pulchra Cogn. como espécie bioindicadora. Tese de doutorado, Universidade de São Paulo, São Paulo.

DOMINGOS, M., KLUMPP, A. \& KLUMPP, G. 1998. Air pollution impact on the Atlantic forest at Cubatão region, Brazil. Ciência e Cultura 50:230-236. 
FURLAN, C.M., SALATINO, A. \& DOMINGOS, M. 1999. Leaf contents of nitrogen and phenolic compounds and their bearing on the herbivore damage to Tibouchina pulchra Cogn. (Melastomataceae), under the influence of air pollutants from industries of Cubatão, São Paulo. Revista Brasileira de Botânica 22:317-323.

GUZY, M.R. \& HEATH, R.L. 1993. Responses to ozone of varieties of common bean (Phaseolus vulgaris L.). New Phytologist 124:617-625.

INOUE, M.T. \& REISSMANN, C.B. 1994. Efeitos da poluição na fotossíntese, dimensões da folha, deposição de particulados e conteúdo de ferro e cobre em alfeneiro (Ligustrum lucidum) da arborização de Curitiba, PR. Floresta 21:3-11.

JAESCHKE, W. 1997. Chemistry module. In Air pollution and vegetation damage in the tropics - the Serra do Mar as an example - Final report 1990-1996 (D. Klockow, H.T. Targa \& W. Vautz, eds.). GKSS-Forschungszentrum Geesthacht GmbH, Geesthacht, p.III.1-III.77.

KELLER, T. \& SCHWAGER, H. 1977. Air pollution and ascorbic acid. European Journal of Forest Pathology 7:338-350.

KLUMPP, A., DOMINGOS, M. \& KLUMPP, G. 1996. Assesment of the vegetation risk by fluoride emissions from fertilisers industries at Cubatão, Brazil. The Science of Total Environment 192:219-228.

KLUMPP, A., DOMINGOS, M., KLUMPP, G. \& GUDERIAN, R. 1997. Vegetation module. In Air pollution and vegetation damage in the tropics - the Serra do Mar as an example Final report 1990-1996 (D. Klockow, H.T. Targa \& W. Vautz, eds.). GKSS-Forschungszentrum Geesthacht GmbH, Geesthacht, p.V.1-V.47.

KLUMPP, A., DOMINGOS, M., MORAES, R.M. \& KLUMPP, G. 1998. Effects of complex air pollution on tree species of the Atlantic rain forest near Cubatão, Brazil. Chemosphere 36:989-994

KLUMPP, A., KLUMPP, G. \& DOMINGOS, M. 1994. Plant as bioindicators of air pollution at the Serra do Mar near the industrial complex of Cubatão, Brazil. Environmental Pollution 85:109-116.

LEA, P.J., ROWLAND-BAMFORD, A.J. \& WOLFENDEN, J. 1996. The effect of air pollution and elevated carbon dioxide on nitrogen metabolism. In Plant response to air pollution (M. Yunus \& M. Iqbal, eds.). John Wiley and Sons, Chichester, p.319-352.

LEITÃO-FILHO, H.F., PAGANO, S.N., CESAR, O., TIMONI, J.L. \& RUEDA, J.J. 1993. Ecologia da Mata Atlântica em Cubatão. Editora da Universidade Estadual Paulista/Universidade Estadual de Campinas, Campinas.

MOONEY, H.A. \& WINNER, W.E. 1988. Carbon gain, allocation, and growth as affected by atmospheric pollutants. In Air pollution and plant metabolism (S. Schulte-Hostede, N.M. Darrall, L.W. Blank \& A.R. Wellburn, eds.). Elsevier Applied Science, London, p.272-287.
PANDEY, J. \& AGRAWAL, M. 1994. Evaluation of air pollution phytotoxicity in a seasonally dry tropical urban environment using three woody perennials. New Phytologist 126:53-61.

PELL, E.J., ECKART, N.A. \& GLICK, R.E. 1994. Biochemical and molecular basis for impairment of photosynthesis potential. Photosynthesis Research 39:453-462.

PFANZ, H., VOLLRATH, B., LOMSKY, B., OPPMANN, B., HYNEK, V., BEYSCHLAG, W. BILGER, W., WHITE, M.V. \& MATERNA, J. 1994. Life expectancy of spruce needles under extremely high air pollution stress: performance of trees in the Ore Mountains. Trees 8:213-222.

REICH, P.B., OLEKSYN, J. \& TJOELKER, M.G. 1994. Relationship of aluminium and calcium to net $\mathrm{CO}_{2}$ exchange among diverse Scots pine provenances under pollution stress in Poland. Oecologia 97:82-92.

RENAUD, J.P., LAITAT, E., MAUFETTE, Y. \& ALLARD, G. 1997. Photoassimilate allocation and photosynthetic and biochemical characteristics of two alfalfa (Medicago sativa L.) cultivars of different ozone sensitivities. Canadian Journal of Botany 76:281-289.

RENNENBERG, H. \& HERSCHBACH, C. 1996. Responses of plants to atmospheric sulphur. In Plant response to air pollution (M. Yunus \& M. Iqbal, eds.). John Wiley and Sons, Chichester, p.285-294.

RENNENBERG, H., HERSCHBACH, C. \& POLLE, A. 1996. Consequences of air pollution on shoot-root interactions. Journal of Plant Physiology 148:296-301.

SAMUELSON, L.J. \& EDWARDS, G.S. 1993. A comparison of sensitivity to ozone in seedlings and trees of Quercus rubra L. New Phytologist 125:373-379.

SCHMIEDEN, U. \& WILD, A. 1995. The contribution of ozone to forest decline. Physiologia Plantarum 94:371-378.

SHAN, Y. 1998. Effects of simulated acid rain on Рinus densiflora: inhibition of net photosynthesis by the pheophytization of chorophyll. Water, Air and Soil Pollution 103:121-127.

SHUGART, L.R. 1994. Biological monitoring. In Contaminants in the environment. A multidisciplinary assessment of risks to man and others organisms (A. Renzoni, N. Mattei, L. Lari \& M.C. Fossi, eds.). Lewis Publishers, New York, p.29-36.

SZABO, A.V. 1999. Conteúdo de enxofre e tióis e parâmetros de crescimento em indivíduos jovens de Tibouchina pulchra Cogn. expostos à poluição aérea, na região do pólo industrial de Cubatão, São Paulo. Dissertação de mestrado, Universidade de São Paulo, São Paulo.

TRESHOW, M. \& ANDERSON, F.K. 1991. Plant stress from air pollution. John Wiley and Sons, Chichester.

ZAGATTO, E.A.G., JACINTHO, A.O., REIS, B.F., KRUG, F.J., BERGAMIN FILHO, H., PESSENDA, L.C.R., MORTATTI, J. \& GINÉ, M.F. 1981. Manual de análise de plantas e águas empregando sistemas de injeção em fluxo. CENA/USP, Piracicaba. 